\section{Determinantes do estado de saúde de crianças ribeirinhas menores de dois anos de idade do Estado do Pará, Brasil: um estudo transversal}

\author{
Health status determinants among river-dwelling \\ children under two years of age in Pará State, \\ Brazil: a cross-sectional study
}

1 Ministério da Saúde, Brasília, Brasil.

2 Universidade Federal do Pará, Belém, Brasil.

Correspondência S. A. Silva

Ministério da Saúde. SAF Sul, Trechos 02, Lotes 05/06, Bloco F, Torre 1,

Edifício Premium, sala 14, Brasília, DF

70070-600, Brasil.

sara.silva@saude.gov.br

\begin{abstract}
The aim of this study was to identify predictors of poor health in children less than two years of age from river-dwelling families in Pará State, Brazil. A total of 202 children were evaluated, considering poor health as the outcome variable, consisting of the combination of nutritional status, psychomotor development, and intercurrent illnesses in the previous month. The data were analyzed with a multilevel hierarchical model, and predictors of poor health were defined as variables with $p<0.05$ after adjustment. According to the crude odds ratio, poor health is associated with families that own their own homes, are older, and present exclusive breastfeeding at two, three, four, and five months. After adjustment, children with families that own their homes showed 2.76 greater odds of having poor health; poor health also increased with age, and was 5.04 higher among children from 18 to 23 months, as compared to infants less than 7 months of age. In these communities, home owning and higher age represent longer exposure to the risk of poor health.
\end{abstract}

Child Health; Rural Population; Nutritional Status; Health Status
Sara Araújo da Silva 1,2 Erly Catarina de Moura 1

\section{Introdução}

A saúde da criança está intimamente relacionada com as condições de morbi-mortalidade nessa fase da vida, demandando ações de prevenção de doenças e promoção de hábitos de vida saudáveis, que, interligados, possibilitem o pleno crescimento e desenvolvimento. Dentre as ações desenvolvidas pelo Ministério da Saúde, destacam-se a atenção ao recém-nascido, a promoção do aleitamento materno, o acompanhamento do crescimento e do desenvolvimento, a imunização e a prevenção e o controle de doenças diarréicas e respiratórias 1 . Essas ações compõem a estrutura básica de cuidados oferecidos pelo sistema de saúde, contribuindo diretamente na redução da morbi-mortalidade 2 .

O crescimento infantil é reconhecido como um importante indicador na área da saúde pública para monitorar o estado de saúde e nutrição de populações ${ }^{3}$, em razão de sua estreita dependência de fatores ambientais. Alimentação, ocorrência de doenças, cuidados gerais e de higiene, condições de habitação e saneamento básico e acesso aos serviços de saúde refletem as condições de vida da criança, no passado e no presente 4 .

A melhoria da saúde infantil está vinculada ao cuidado integrado. Propostas de promoção da saúde e prevenção de doenças só podem ser estipuladas a partir do conhecimento prévio do quadro situacional do grupo ou da comunidade na qual a criança se insere, a fim de propor estra- 
tégias que possibilitem melhorias das condições de vida.

A situação de saúde sofre influência direta do estado nutricional, do desenvolvimento físicomotor e de intercorrências na saúde, portanto a melhoria da situação de saúde infantil resulta da diminuição de taxas de mortalidade e desnutrição 1. Em 2007, informações do Departamento de Informática do SUS (http://www.datasus.gov. br, acessado em 01/Set/2009) apontaram que aproximadamente $3 \%$ das mortes em crianças brasileiras, nos dois primeiros anos de vida, tinham sido causadas por doenças respiratórias, infecciosas e parasitárias. Além disso, é preocupante a situação nutricional de crianças menores de dois anos: $12,3 \% 4$ destas encontram-se em quadro de desnutrição, quando avaliadas pelo índice altura para idade, o qual expressa o crescimento linear da criança, ou seja, o estado nutricional desde o nascimento até o momento atual. A vulnerabilidade nessa fase da vida, associada ao estado nutricional e às doenças, está intimamente relacionada ao desenvolvimento físico-motor, definindo, assim, o estado de saúde de crianças.

Sabendo que são escassos os estudos sobre o estado de saúde de crianças ribeirinhas no país, este estudo tem como objetivo identificar os determinantes da saúde de crianças menores de dois anos residentes em populações ribeirinhas do Estado do Pará.

\section{Metodologia}

A população deste estudo foram os menores de dois anos de idade residentes em comunidades ribeirinhas do Estado do Pará. Dados pontuais de algumas comunidades sugerem que a população ribeirinha do estado seja de 40 mil famílias. Por se desconhecer o tamanho real da população estudada, uma vez que as áreas rurais da Região Norte do Brasil nunca foram avaliadas nos censos demográficos anteriores, optou-se pelo cálculo conservador do tamanho amostral. Considerouse proporção de saúde ruim na população igual a $50 \%$ e nível de significância de $5 \%$, com precisão absoluta aproximada de $8 \%$, o que resultou num tamanho amostral de 200 crianças.

Dessa forma, a seleção da população estudada foi realizada em três estágios: (1) sorteio de quatro municípios (conforme distribuição geográfica), (2) escolha das comunidades (contemplando as diversidades culturais e de acesso) e (3) eleição das crianças (universal, sempre que possível, e aleatória, quando não universal, num mínimo de quarenta por comunidade). A amostra do estado contemplou quatro municí- pios: Aveiro (região sudoeste), Barcarena (região metropolitana), Cametá (região nordeste) e Santarém (região do baixo Amazonas). Inicialmente, em cada município, procurou-se localizar as populações ribeirinhas com o apoio do Centro Regional da Secretaria Estadual de Saúde e da Secretaria Municipal de Saúde. Cumpre salientar que não necessariamente as comunidades investigadas representam a comunidade ribeirinha do Estado do Pará, por falta de dados demográficos que permitam avaliar a representatividade da população estudada para o universo das populações ribeirinhas.

Foi estudada uma comunidade ribeirinha de cada município. Assim, a comunidade de Aveiro foi selecionada por representar o centro do município e viver principalmente da agricultura; a de Barcarena, por ser residente em uma ilha próxima ao centro do município, de fácil acesso, que vive principalmente da pesca - a ilha de Trambioca; a de Cametá, por ser uma comunidade distante do centro do município e de difícil acesso, que vive principalmente da agricultura - a Vila Torres; a de Santarém, por ser de origem indígena, que vive principalmente do turismo Alter do Chão.

Cada família (composta por todos os moradores da residência, independente do grau de parentesco) foi convidada a participar do estudo. Aplicou-se questionário para identificação da composição familiar, da situação de moradia e de formas de assistência à saúde. A todos os responsáveis pelas crianças menores de dois anos de idade foi aplicado um questionário complementar com dados sobre antecedentes gestacionais maternos, condições de nascimento, características de aleitamento materno, situação de imunização, desenvolvimento físicomotor, assistência à saúde, intercorrências no último mês, com foco para as doenças diarréicas e respiratórias. Cada criança foi submetida à tomada de peso e comprimento para classificação do estado nutricional. O peso foi obtido em libras, em balança digital (Sunbeam, Wichita, Estados Unidos), e transformado para quilogramas (kg), multiplicando-se o valor obtido por 0,45 . O comprimento foi medido em centímetros $(\mathrm{cm})$ usando estadiômetro portátil (Alturexata, Belo Horizonte, Brasil).

A variável desfecho foi o estado de saúde, classificado em ruim e não ruim, conforme combinação das variáveis estado nutricional da criança, desenvolvimento físico-motor e intercorrências na saúde nos últimos trinta dias.

$\mathrm{O}$ estado nutricional, peso e estatura foram classificados conforme os valores de $\mathrm{z}$ escore 5 , considerando-se deficiência para valores menores do que -1. Atribuiu-se valor 0 para ausência 
de deficiência de peso para idade e de deficiência de estatura para idade; 1 para presença de deficiência de peso para idade ou de deficiência de estatura para idade; 2 para presença de deficiência de peso para idade e de deficiência de estatura para idade.

O desenvolvimento físico-motor foi classificado considerando-se a capacidade da criança em realizar tarefas conforme a idade, permitindo avaliar marcos da maturidade neurológica e do desenvolvimento infantil ${ }^{6}$. Atribuiu-se valor 0 para adequado e 1 para atrasado.

Para a classificação das intercorrências na saúde da criança, considerou-se a freqüência de eventos de doenças nos últimos trinta dias, sendo 0 para nenhuma ocorrência no último mês e 1 para alguma ocorrência nesse período. Portanto, se a criança tivesse apenas uma ocorrência no ultimo mês, nos últimos 15 dias ou no dia da entrevista, receberia valor 1 para cada evento, variando de 1 até 3 . O valor foi multiplicado pelo número de ocorrências relatadas, cujo produto variou de 0 a 6 . Assim, a soma dos escores desses três indicadores variou de 0 a 9 , sendo considerado, para a classificação do estado de saúde, o corte em saúde não ruim $(<3)$ e saúde ruim $(\geq 3)$.

Foram consideradas independentes as características sócio-demográficas das famílias e das mães das crianças, condições de moradia, assistência à saúde das famílias, antecedentes gestacionais maternos, condições de nascimento, características demográficas das crianças, probabilidade de aleitamento materno exclusivo em seis diferentes momentos - com um, dois, três, quatro, cinco e seis meses de idade - e condições de assistência à saúde das crianças.

As características sócio-demográficas foram divididas em dois blocos: família e mãe da criança. No primeiro bloco, considerou-se idade (17-35 ou 36-83 anos de idade) e escolaridade do chefe da família (0-4 ou 5-16 anos de estudo), sua inserção no mercado de trabalho (não ou sim), número de moradores (3-5 ou 6-18), número de adultos (1-2 ou $\geq 3$ ) e número de crianças (1-2 ou $\geq 3$ ) por unidade domiciliar. No segundo bloco, levantaram-se idade (15-22 ou 23-43 anos de idade) e escolaridade da mãe (0-7 ou 8-15 anos de estudo), sua inserção no mercado de trabalho e estabilidade da união conjugal (não ou sim).

As condições de moradia foram avaliadas conforme posse (não própria ou própria), densidade familiar no domicílio $(<2$ ou $\geq 2)$ e condições de saneamento básico (inadequado ou adequado). Este foi considerado adequado quando havia tratamento da água para beber, destino adequado para o esgoto e correto acondicionamento do lixo no domicílio. Adicionalmente, para o conjunto das crianças estudadas, levou-se em conta a variável localização da moradia (Aveiro, Barcarena, Cametá ou Santarém).

A assistência à saúde da família foi avaliada por meio das seguintes variáveis: visita do agente comunitário de saúde, visita da equipe do Programa Saúde da Família - categorizadas em sim, $\geq 1$ visita por mês; sim, < 1 visita por mês; ou não recebe visita - e local de assistência à saúde da família - em geral, classificado em unidade local ou unidade externa.

Variáveis referentes aos antecedentes gestacionais maternos, como realização de pré-natal, doenças maternas e tabagismo durante a gravidez foram categorizadas em não ou sim. A idade gestacional da primeira consulta no pré-natal foi classificada em três categorias: $<3$ meses, $\geq 3$ meses, ou não fez pré-natal; o número de consultas realizadas no pré-natal foi classificado em inadequado $(<7$ consultas $)$ ou adequado $(\geq 7$ consultas).

Para a descrição das condições de nascimento das crianças foram utilizados local de nascimento (hospital ou domicílio), tipo de parto (normal/fórceps ou cirúrgico), maturidade gestacional conforme prematuridade obtida do cartão de saúde da criança (não ou sim) e baixo peso ao nascer quando inferior a $2.500 \mathrm{~g}$ (não ou sim). As características demográficas das crianças foram categorizadas conforme sexo (masculino ou feminino) e idade $(0-5,6-11,12-17$ ou $18-23$ meses).

Quanto às características de aleitamento, foi considerada a probabilidade de aleitamento materno exclusivo (não ou sim) em seis diferentes momentos, sendo estes com um mês de idade, dois, três, quatro, cinco e seis meses de idade. Foi considerado aleitamento materno exclusivo no primeiro mês para as crianças com pelo menos um mês de vida e que não haviam recebido outro alimento até essa idade; para aquelas que referiram ter recebido outro alimento antes de um mês, não foi considerado exclusivo. Das 202 crianças estudadas, 11 ainda não haviam completado um mês e estavam em aleitamento materno exclusivo. Para estes casos, calculou-se a chance de permanência ou não de aleitamento materno exclusivo até completarem um mês, levando-se em conta o comportamento das demais crianças da mesma comunidade. Para os demais meses, seguiu-se o mesmo procedimento, atribuindo-se valores para 20 crianças aos dois meses, 24 crianças aos três meses, 37 aos quatro meses, 41 aos cinco meses e 43 aos seis meses. Além da localidade, as variáveis utilizadas para determinar essa chance foram idade, peso ao nascer e inserção da mãe no mercado de trabalho. Desse modo, a probabilidade de aleitamento materno foi de $95,5 \%$ no primeiro mês de vida, $86,1 \%$ no segun- 
do, $78,2 \%$ no terceiro, $64,4 \%$ no quarto, $57,9 \%$ no quinto e 50,5\% no sexto mês. As condições de assistência à saúde da criança foram avaliadas de acordo com a situação de imunização (inadequada ou adequada), considerando-se adequados para os casos em que as vacinas haviam sido realizadas nas datas aprazadas no cartão da criança; local de acompanhamento (nenhum, unidade local ou unidade externa); registro da curva de crescimento no cartão da criança (não ou sim); idade da primeira consulta (inadequada, se $>1$ mês, ou adequada, se $\leq 1$ mês) e concentração de consultas ( $<0,5$ ou $\geq 0,5$ por mês de idade).

Neste estudo, foi utilizado modelo multinível, que permite levar em conta a estrutura hierárquica dos dados e variáveis contextuais, considerando-se a distância entre as variáveis e o desfecho. Essa análise permite a entrada das variáveis explanatórias no modelo numa ordem previamente especificada e baseada em um modelo conceitual de relação lógica ou teórica entre as variáveis 7 .

No primeiro nível, foram consideradas as variáveis sócio-demográficas familiares e maternas (mais distais); no segundo, as condições de moradia e de assistência à saúde da família; no terceiro, os antecedentes gestacionais maternos; no quarto, as condições de nascimento, características demográficas da criança e de aleitamento materno; no quinto as condições de assistência à saúde da criança, mais proximais, conforme ilustra a Figura 1.

Calculou-se a prevalência de estado de saúde ruim, considerando-se intervalo de 95\% de confiança (IC95\%), segundo as variáveis explanatórias. Aquelas associadas ao estado de saúde ruim com $\mathrm{p}<0,05$, conforme teste do qui-quadrado, foram selecionadas para análise multivariada por regressão logística.

Para identificar as variáveis associadas à saúde ruim, calculou-se primeiramente a razão de chance (RC) bruta para todas as variáveis com $\mathrm{p}<$ 0,05 pelo teste do qui-quadrado; em seguida a razão de chance foi ajustada para todas as variáveis que apresentaram $\mathrm{p}<0,20$ na análise bivariada, iniciando-se pelo nível hierárquico mais distal até o mais proximal. Foram consideradas preditoras da saúde ruim as variáveis que se mantiveram associadas com $\mathrm{p}<0,05$ após ajuste.

As análises de dados foram realizadas utilizando-se os aplicativos Epi Info versão 3.5.1 (Centers for Disease Control and Prevention, Atlanta, Estados Unidos), SPSS versão 13.0 (SPSS Inc., Chicago, Estados Unidos), Excel (Microsoft, Estados Unidos) e WHO Anthro 2005 (World Health Organization, Genebra, Suíça).

Este estudo está contemplado no projeto de pesquisa Determinantes da Saúde Infantil de Po- pulações Ribeirinhas do Pará, aprovado pelo Comitê de Ética em Pesquisa para Seres Humanos do Centro de Ciências da Saúde da Universidade Federal do Pará, e contou com apoio financeiro da Secretaria de Ciência, Tecnologia e Insumos Estratégicos do Pará (SECTAM/PA), por meio da Fundação de Amparo à Pesquisa do Estado do Pará (FAPESPA).

\section{Resultados}

Das 202 crianças avaliadas, 59,4\% apresentaram estado de saúde ruim. Dentre os componentes utilizados para avaliar a saúde ruim, observou-se que o estado nutricional se distribuiu em 53,5\% das crianças sem deficiência de peso e estatura para idade; $11,9 \%$ de deficiência de peso para idade; $17,3 \%$ de deficiência de estatura para idade; $17,3 \%$ de deficiência de peso e de estatura para idade. Observou-se ainda que o desenvolvimento físico-motor foi adequado em $97 \%$ dos casos e inadequado em 3\%; que as intercorrências atingiram $34,2 \%$ das crianças no dia da entrevista; $42,1 \%$ nos 15 dias que antecederam a entrevista; $17,8 \%$ entre $16-30$ dias antes da entrevista e que apenas 5,9\% das crianças não apresentaram nenhuma intercorrência nos últimos trinta dias (dados não mostrados).

A Tabela 1 apresenta a prevalência de saúde ruim segundo características sócio-demográficas das famílias e das mães das crianças e condições de moradia. Encontrou-se associação, no nível de $\mathrm{p}<0,20$, entre saúde ruim e idade do chefe da família, inserção deste no mercado de trabalho e posse da moradia.

No que se refere a assistência à saúde das famílias, antecedentes gestacionais maternos e condições de nascimento da criança, apresentaram associação $(\mathrm{p}<0,20)$ com estado de saúde ruim as seguintes variáveis: realização de prénatal, idade gestacional da primeira consulta no pré-natal e tabagismo materno durante a gestação (Tabela 2).

A Tabela 3 mostra uma tendência crescente do estado de saúde ruim com o aumento da idade da criança, com a redução da probabilidade de aleitamento materno exclusivo e com concentração de consultas por mês.

A Tabela 4 apresenta as RC, bruta e ajustada, do estado de saúde ruim das crianças avaliadas; as variáveis independentes foram introduzidas conforme o nível, no modelo de análise hierárquica, utilizando regressão logística. Observa-se que a RC bruta para saúde ruim é maior para as crianças de famílias que residem em casa própria, que apresentam maior idade e que têm probabilidade de aleitamento materno exclusivo aos 
Figura 1

Modelo teórico hierarquizado para avaliação do estado de saúde da criança.

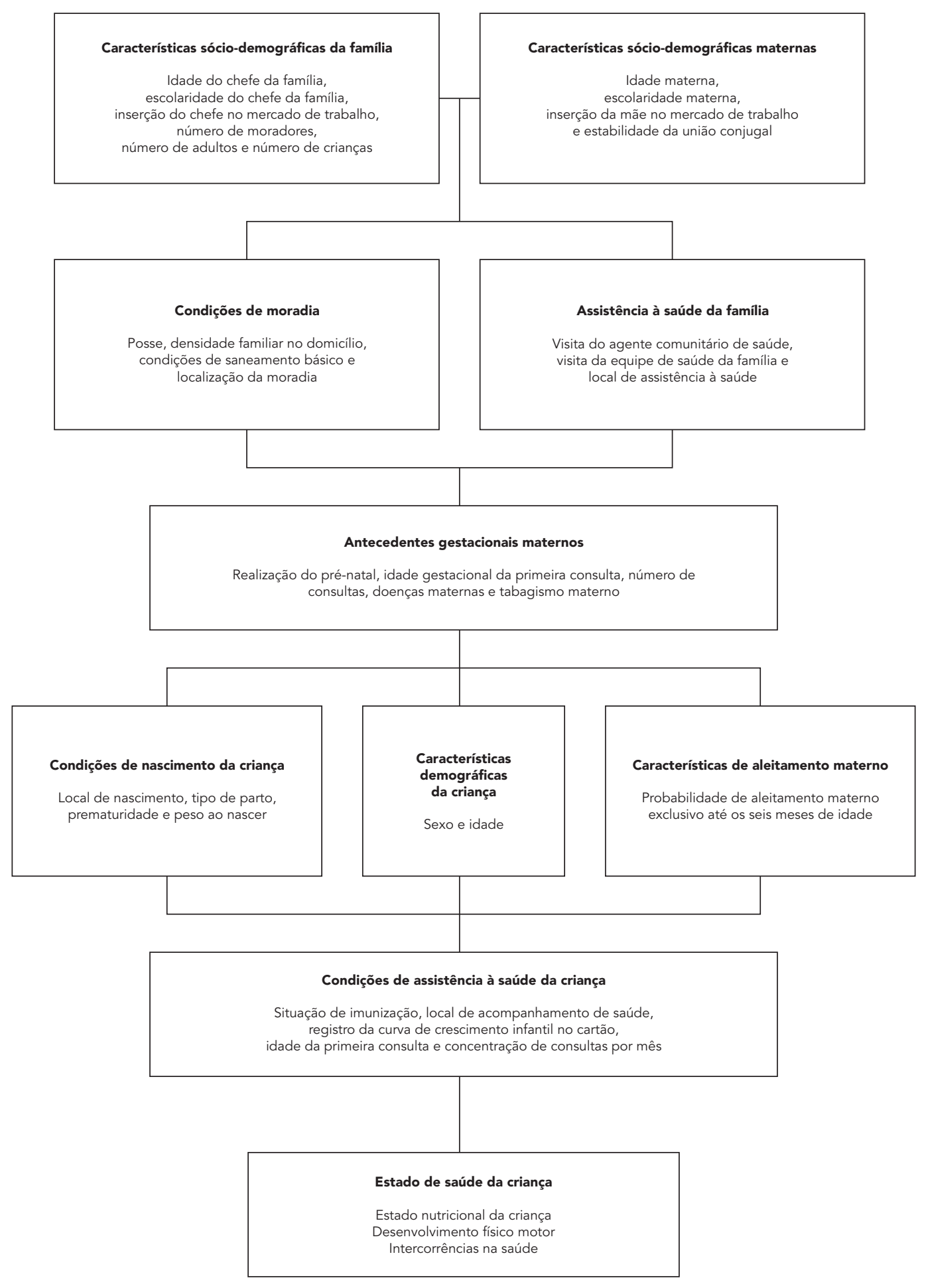


Tabela 1

Prevalência (\%) e intervalo de 95\% de confiança (IC95\%) de saúde ruim segundo características sócio-demográficas e condições de moradia das famílias e das mães de crianças ribeirinhas. Pará, Brasil, 2008.

\begin{tabular}{|c|c|c|c|c|}
\hline Características & $\mathbf{n}$ & $\%$ & IC95\% & Valor de $p$ \\
\hline Idade do chefe da família (anos) & & & & 0,086 \\
\hline $17-35$ & 101 & 65,3 & $55,9-74,8$ & \\
\hline $36-83$ & 101 & 53,5 & $43,6-63,4$ & \\
\hline Escolaridade do chefe da família (anos) & & & & 0,939 \\
\hline $0-4$ & 77 & 59,7 & $48,5-70,9$ & \\
\hline $5-16$ & 125 & 59,2 & $50,5-67,9$ & \\
\hline Inserção do chefe da família no mercado de trabalho & & & & 0,160 \\
\hline Não & 113 & 63,7 & $54,7-72,7$ & \\
\hline Sim & 89 & 53,9 & $43,4-64,5$ & \\
\hline Número de moradores & & & & 0,749 \\
\hline $3-5$ & 84 & 60,7 & $50,1-71,4$ & \\
\hline $6-8$ & 118 & 58,5 & $49,5-67,5$ & \\
\hline Número de adultos & & & & 0,502 \\
\hline $1-2$ & 119 & 61,3 & $52,5-70,2$ & \\
\hline$\geq 3$ & 83 & 56,6 & $45,7-67,5$ & \\
\hline Número de crianças & & & & 0,991 \\
\hline $1-2$ & 74 & 59,5 & $48,0-70,9$ & \\
\hline$\geq 3$ & 128 & 59,4 & $50,8-68,0$ & \\
\hline Idade da mãe (anos) & & & & 0,324 \\
\hline $15-22$ & 95 & 55,8 & $45,6-66,0$ & \\
\hline $23-43$ & 107 & 62,6 & $53,3-71,9$ & \\
\hline Escolaridade da mãe (anos) & & & & 0,356 \\
\hline $0-7$ & 98 & 56,1 & $46,1-66,1$ & \\
\hline $8-15$ & 104 & 62,5 & $53,0-72,0$ & \\
\hline Inserção da mãe no mercado de trabalho & & & & 0,801 \\
\hline Não & 126 & 58,7 & $50,0-67,4$ & \\
\hline Sim & 76 & 60,5 & $49,3-71,8$ & \\
\hline Estabilidade da união conjugal & & & & 0,610 \\
\hline Não & 43 & 62,8 & $47,7-77,8$ & \\
\hline Sim & 159 & 58,5 & $50,7-66,2$ & \\
\hline Localização de moradia & & & & 0,353 \\
\hline Aveiro & 56 & 64,3 & $51,3-77,2$ & \\
\hline Santarém & 54 & 63,0 & $49,7-76,3$ & \\
\hline Cametá & 42 & 47,6 & $31,9-63,4$ & \\
\hline Barcarena & 50 & 60,0 & $45,9-74,1$ & \\
\hline Posse & & & & 0,035 \\
\hline Não própria & 25 & 40,0 & $19,4-60,6$ & \\
\hline Própria & 177 & 62,1 & $54,9-69,4$ & \\
\hline Densidade familiar no domicílio & & & & 0,465 \\
\hline $0,5-1,86$ & 90 & 62,2 & $52,0-72,4$ & \\
\hline $2,0-12,0$ & 112 & 57,1 & $47,8-66,5$ & \\
\hline Condições de saneamento básico & & & & 0,979 \\
\hline Inadequado & 116 & 59,5 & $50,4-68,5$ & \\
\hline Adequado & 86 & 59,3 & $48,7-69,9$ & \\
\hline Estado de saúde ruim & 120 & 59,4 & $52,6-66,2$ & - \\
\hline
\end{tabular}


Tabela 2

Prevalência (\%) e intervalo de 95\% de confiança (IC95\%) de saúde ruim segundo assistência à saúde de famílias ribeirinhas, antecedentes gestacionais maternos e condições de nascimento de crianças ribeirinhas menores de dois anos de idade. Pará, Brasil, 2008.

\begin{tabular}{|c|c|c|c|c|}
\hline Características & $\mathbf{n}$ & $\%$ & IC95\% & Valor de $p$ \\
\hline Visita do agente comunitário de saúde * & & & & 0,870 \\
\hline $\operatorname{Sim}$ ( $\geq 1$ visita/mês) & 139 & 63,6 & $41,8-85,5$ & \\
\hline Sim (< 1 visita/mês) & 22 & 61,5 & $45,6-77,5$ & \\
\hline Não recebe visita & 39 & 58,3 & $50,0-66,6$ & \\
\hline Visita da equipe do Programa Saúde da Família ** & & & & 0,369 \\
\hline $\operatorname{Sim}(\geq 1$ visita/mês) & 22 & 45,5 & $22,9-68,1$ & \\
\hline $\operatorname{Sim}(<1$ visita/mês) & 18 & 61,1 & $36,2-86,1$ & \\
\hline Não recebe visita & 161 & 60,9 & $53,2-68,5$ & \\
\hline 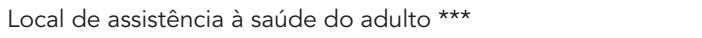 & & & & 0,423 \\
\hline Unidade local & 130 & 62,3 & $53,9-70,7$ & \\
\hline Unidade externa & 63 & 54,0 & $41,3-66,6$ & \\
\hline Realização de pré-natal & & & & 0,043 \\
\hline Não & 8 & 25,0 & $0,0-63,7$ & \\
\hline Sim & 194 & 60,8 & $53,9-67,8$ & \\
\hline Idade gestacional (semanas) da primeira consulta no pré-natal & & & & 0,121 \\
\hline Adequado $(<3)$ & 113 & 61,9 & $52,9-71,0$ & \\
\hline Inadequado (3-6) & 81 & 59,3 & $48,3-70,2$ & \\
\hline Não fez pré-natal & 8 & 25,0 & $0,0-63,7$ & \\
\hline Número de consultas & & & & 0,932 \\
\hline Inadequada (0-6) & 146 & 59,6 & $51,5-67,6$ & \\
\hline Adequada $(\geq 7)$ & 56 & 58,9 & $45,6-72,2$ & \\
\hline Doenças maternas & & & & 0,496 \\
\hline Não & 145 & 57,9 & $49,8-66,1$ & \\
\hline Sim & 57 & 63,2 & $50,2-76,1$ & \\
\hline Tabagismo materno & & & & 0,184 \\
\hline Não & 189 & 58,2 & $51,1-65,3$ & \\
\hline Sim & 13 & 76,9 & $50,4-100,0$ & \\
\hline Local de nascimento & & & & 0,876 \\
\hline Hospital & 164 & 59,1 & $51,5-66,7$ & \\
\hline Domicílio & 38 & 60,5 & $44,2-76,8$ & \\
\hline Tipo de parto & & & & 0,818 \\
\hline Normal/Fórceps & 166 & 59,0 & $51,5-66,6$ & \\
\hline Cirúrgico & 36 & 61,1 & $44,4-77,8$ & \\
\hline Prematuridade & & & & 0,856 \\
\hline Não & 194 & 59,3 & $52,3-66,3$ & \\
\hline Sim & 8 & 62,5 & $19,2-105,8$ & \\
\hline Baixo peso ao nascer & & & & 0,360 \\
\hline Não & 177 & 57,6 & $50,3-65,0$ & \\
\hline Sim & 16 & 75,0 & $51,2-98,8$ & \\
\hline Não sabe & 9 & 66,7 & $28,2-100,0$ & \\
\hline
\end{tabular}

* Dois casos não souberam informar;

** Um caso não soube informar;

$\star \star \star$ Nove casos não souberam informar. 
Tabela 3

Prevalência (\%) e intervalo de 95\% de confiança (IC95\%) de saúde ruim segundo características demográficas, probabilidade de aleitamento materno exclusivo e condições de assistência à saúde de crianças ribeirinhas menores de dois anos de idade. Pará, Brasil, 2008.

\begin{tabular}{|c|c|c|c|c|}
\hline Características & $\mathbf{n}$ & $\%$ & IC95\% & Valor de $p$ \\
\hline Sexo & & & & 0,324 \\
\hline Masculino & 95 & 55,8 & $45,6-66,0$ & \\
\hline Feminino & 107 & 62,6 & $53,3-71,9$ & \\
\hline Idade (meses) & & & & $<0,001$ \\
\hline $0-5$ & 64 & 39,1 & $26,8-51,3$ & \\
\hline $6-11$ & 55 & 58,2 & $44,7-71,6$ & \\
\hline $12-17$ & 46 & 73,9 & $60,7-87,1$ & \\
\hline $18-23$ & 37 & 78,4 & $64,5-92,3$ & \\
\hline \multicolumn{5}{|l|}{ Probabilidade de aleitamento materno } \\
\hline Com 1 mês & 193 & 60,1 & $53,1-67,1$ & 0,350 \\
\hline Com 2 meses & 174 & 63,2 & $56,0-70,5$ & 0,006 \\
\hline Com 3 meses & 158 & 64,6 & $57,0-72,1$ & 0,005 \\
\hline Com 4 meses & 130 & 66,9 & $58,7-75,1$ & 0,003 \\
\hline Com 5 meses & 117 & 65,8 & $57,1-74,5$ & 0,030 \\
\hline Com 6 meses & 90 & 67,8 & $57,9-77,6$ & 0,066 \\
\hline Imunização & & & & 0,313 \\
\hline Inadequada & 33 & 51,5 & $33,5-69,5$ & \\
\hline Adequada & 169 & 60,9 & $53,5-68,4$ & \\
\hline Realiza acompanhamento de saúde & & & & 0,294 \\
\hline Sim (unidade local) & 99 & 63,6 & $54,0-73,3$ & \\
\hline Sim (unidade externa) & 68 & 58,8 & $46,8-70,8$ & \\
\hline Não realiza & 35 & 48,6 & $31,2-66,0$ & \\
\hline Registro da curva de crescimento no cartão & & & & 0,931 \\
\hline Não & 125 & 58,4 & $49,6-67,2$ & \\
\hline Sim & 67 & 61,2 & $49,2-73,2$ & \\
\hline Não sabe & 10 & 60,0 & $23,1-96,9$ & \\
\hline Idade da primeira consulta (meses) & & & & 0,454 \\
\hline Inadequada (> 1) & 64 & 65,6 & $53,7-77,6$ & \\
\hline Adequada $(<1)$ & 58 & 55,2 & $42,0-68,4$ & \\
\hline Não sabe & 80 & 57,5 & $46,4-68,6$ & \\
\hline Concentração de consultas por mês & & & & 0,080 \\
\hline$<0,5$ & 85 & 68,2 & $58,1-78,3$ & \\
\hline$\geq 0,5$ & 65 & 50,8 & $38,3-63,3$ & \\
\hline Não sabe & 52 & 55,8 & $41,8-69,7$ & \\
\hline
\end{tabular}

dois, três, quatro e cinco meses. Após ajuste, para todas as variáveis com $\mathrm{p}<0,20$, a associação entre saúde ruim e probabilidade de aleitamento materno exclusivo desaparece, permanecendo apenas a associação com posse da moradia e idade da criança. As crianças provenientes de famílias com casa própria têm 2,76 vezes mais chance de ter saúde ruim do que aquelas sem casa própria. A chance de saúde ruim aumenta com a idade, chegando a 5,04 vezes mais naquelas entre 18-23 meses, comparativamente às crianças entre 0-5 meses.

\section{Discussão}

Este estudo mostrou que $59,4 \%$ das crianças menores de dois anos de idade foram consideradas com saúde ruim, não havendo diferença entre as comunidades avaliadas. Embora os achados da presente pesquisa possam ser representativos para as comunidades avaliadas e outras semelhantes, considerando-se erro amostral de 6,8\% e coeficiente de $95 \%$ de confiança, não se pode inferir os dados encontrados para as comunidades ribeirinhas do Estado do Pará como um todo. A maioria das pesquisas brasileiras sobre saúde de 
Razão de chance (RC) bruta e ajustada do estado de saúde ruim de crianças ribeirinhas menores de dois anos de idade. Pará, Brasil, 2008.

\begin{tabular}{|c|c|c|c|c|c|c|}
\hline Variáveis & RC bruta & IC95\% & Valor de $p$ & RC ajustada * & IC95\% & Valor de $p$ \\
\hline Posse & & & 0,039 & & & 0,034 \\
\hline Não própria & 1,00 & & & 1,00 & & \\
\hline Própria & 2,46 & $1,05-5,80$ & & 2,76 & $1,07-7,08$ & \\
\hline Realização de pré-natal & & & 0,064 & & & 0,213 \\
\hline Não & 1,00 & & & 1,00 & & \\
\hline Sim & 4,66 & $0,92-23,68$ & & 3,17 & $0,53-19,09$ & \\
\hline Idade (meses) & & & $<0,001$ & & & $<0,001$ \\
\hline $0-5$ & 1,00 & & & 1,00 & & \\
\hline $6-11$ & 2,17 & $1,04-4,52$ & & 1,55 & $0,64-3,77$ & \\
\hline $12-17$ & 4,42 & $1,93-10,11$ & & 4,42 & $1,61-12,15$ & \\
\hline $18-23$ & 5,65 & $2,23-14,33$ & & 5,04 & $1,70-14,94$ & \\
\hline \multicolumn{7}{|c|}{ Probabilidade de aleitamento materno } \\
\hline Com 2 meses & & & 0,008 & & & 0,406 \\
\hline Não & 1,00 & & & 1,00 & & \\
\hline Sim & 3,09 & $1,35-7,11$ & & 1,93 & $0,48-7,73$ & \\
\hline Com 3 meses & & & 0,006 & & & 0,783 \\
\hline Não & 1,00 & & & 1,00 & & \\
\hline Sim & 2,63 & $1,33-5,21$ & & 1,18 & $0,32-4,40$ & \\
\hline Com 4 meses & & & 0,004 & & & 0,803 \\
\hline Não & 1,00 & & & 1,00 & & \\
\hline Sim & 2,39 & 1,33-4,31 & & 1,22 & $0,32-4,65$ & \\
\hline Com 5 meses & & & 0,030 & & & 0,840 \\
\hline Não & 1,00 & & & 1,00 & & \\
\hline Sim & 1,89 & $1,06-3,33$ & & 0,76 & $0,23-2,52$ & \\
\hline Com 6 meses & & & 0,067 & & & 0,372 \\
\hline Não & 1,00 & $0,96-2,99$ & & 1,00 & $0,16-2,08$ & \\
\hline Sim & 1,69 & & & 0,58 & & \\
\hline
\end{tabular}

IC95\%: intervalo de 95\% de confiança.

* Ajustado para idade do chefe de família, inserção deste no mercado de trabalho, posse, realização de pré-natal, idade gestacional da primeira consulta no pré-natal, tabagismo materno, idade da criança, probabilidade de aleitamento materno com dois, três, quatro e cinco meses de vida e concentração de consultas da criança por mês.

crianças se refere a alguma característica específica, como o estado nutricional ou a ocorrência de determinadas doenças, em geral as diarréicas e/ou respiratórias, mas nenhuma delas utiliza indicador composto para definir estado de saúde, da forma como se apresentou neste trabalho (estado nutricional + desenvolvimento físico-motor + intercorrência).

Monteiro et al. 8, avaliando uma amostra de crianças menores de cinco anos estudadas na Pesquisa Nacional de Demografia e Saúde da Criança e da Mulher (PNDS 2006) 9 , mas excluindo aquelas da área rural da Região Norte, observaram deficiência de estatura para idade em $6,8 \%$ e de peso para estatura em $1,6 \%$. Os autores utilizaram $\mathrm{z}$ escore $<-2$, conforme referência da Organização Mundial da Saúde (OMS) de 2004 10, verificando maior deficiência entre as crianças com mães de baixa escolaridade.

Engstrom \& Anjos 11 apontaram que 7,2\% entre os menores de seis meses e $14,3 \%$ daqueles entre seis meses e dois anos de idade apresentavam deficiência de estatura para idade, segundo dados da Pesquisa Nacional sobre Saúde e Nutrição (PNSN 1989) 12; a pesquisa utilizou o ponto de corte de $\mathrm{z}$ escore $(<-2)$, com base na referência do National Center for Health Statistics (NCHS) 13, e também não avaliou crianças da área rural da Região Norte. No nosso estudo, utilizando o ponto de corte em z escore $(<-2)$, identificamos $13,4 \%$ de deficiência de estatura para idade e $5,4 \%$ de peso para estatura em menores de dois anos; $6,3 \%$ e $16,7 \%$ de deficiência de estatura para idade respectivamente entre os 
menores de seis meses e entre aqueles com seis meses a dois anos. Os dados não podem ser diretamente comparados, pois a população deste estudo é diferente, caracterizando-se muito mais como da área rural quando comparada à dos dois estudos citados; além disso, esta investigação desenvolveu-se em período bastante diferente do segundo. Ainda assim, nossos dados apresentam maiores prevalências de deficiência em relação ao primeiro estudo, conforme esperado para as comunidades estudadas.

Para a avaliação do desenvolvimento físicomotor de crianças, a literatura aponta estudos observacionais de associação entre desenvolvimento motor global e habilidades orais 13 , ou identificação de fatores de risco que podem interferir no curso normal de desenvolvimento ${ }^{14}$, entretanto são pesquisas de acompanhamento com um grupo pequeno de crianças, com dois anos de idade ou menos. O presente estudo questionou mães e/ou cuidadores sobre a capacidade da criança em realizar tarefas conforme a idade. Com isso, foi possível avaliar marcos da maturidade neurológica e do desenvolvimento infantil segundo parâmetros da OMS 5, identificando-se que $97 \%$ apresentavam adequado desenvolvimento físico-motor.

A PNDS ${ }^{9}$ aponta que, na Região Norte, $14,6 \%$ das crianças menores de cinco anos apresentaram diarréia nos últimos 15 dias; dentre estas, os valores encontrados foram de 4,8\%, $11,2 \%$ e $16,3 \%$ entre os menores de seis meses, entre 6-11 meses e entre 12-23 meses respectivamente. Analisando a freqüência de diarréia, nosso estudo mostra $18,5 \%, 14,3 \%$ e $32,6 \%$ nas respectivas faixas etárias, valores preocupantes por representarem o triplo nos mais jovens e o dobro naqueles no segundo ano de vida.

Estudo de base populacional realizado em uma amostra de 775 crianças de 0-59 meses na cidade de Rio Grande (Rio Grande do Sul) 15 identificou que 23,9\% apresentavam doenças respiratórias. No nosso estudo, identificamos $47,5 \%$ das crianças acometidas por doenças respiratórias no último mês, valor muito maior do que o apontado no estudo supracitado.

Ambas as situações, doenças diarréicas e respiratórias, podem refletir a falta de acesso aos serviços de saúde, muitas vezes limitado à visita do agente de saúde: $25,4 \%$ das crianças com diarréia nos últimos 15 dias e $25 \%$ das crianças com doenças respiratórias nos últimos trinta dias referiram não receber visita do agente sanitário, contra $16,8 \%$ e $14,2 \%$, respectivamente, para as crianças que não relataram essas doenças nos mesmos períodos.

Os dados da literatura não apresentam a situação de populações de áreas rurais da Região
Norte e, em geral, realizam avaliações pontuais de apenas algumas características que compuseram o indicador avaliado neste estudo, fator de suma importância para o planejamento de ações e estratégias em saúde. Reconhecidamente, é incipiente a análise da situação de saúde de populações ribeirinhas, que, segundo Guarim 16, caracterizam-se por se localizarem às margens de rios e desenvolverem, permanentemente, uma estreita relação com o ambiente, no que se refere à conservação do solo, da água, da fauna e da flora, constituindo, assim, uma condição sóciocultural típica de comunidades tradicionais.

O presente estudo não avaliou especificamente as condições associadas com o acometimento de doenças nas crianças, entretanto esta é uma das características contempladas no desfecho em questão e, segundo a literatura, crianças que vivem em domicílios com precárias condições de piso e teto, com ausência de canalização para água, sem sanitários com descarga e sem geladeira apresentam maior ocorrência de diarréia, sendo a ausência de água encanada o mais importante fator associado à doença ${ }^{17}$. Quanto ao saneamento básico, observamos que $57,4 \%$ das crianças residiam em condições inadequadas. Demais estudos sugerem que melhor distribuição de renda e acesso à escola, principalmente pelas mães, reduziriam os eventos de doenças respiratórias, mediante melhoria das condições de moradia e ambiente familiar ${ }^{18}$. Nesta pesquisa, $48,5 \%$ das mães das crianças estudaram menos do que oito anos.

A maioria das investigações justifica a eleição do estado nutricional como proxy do estado de saúde 3 , pois a saúde da criança está intimamente relacionada com as condições de morbimortalidade, como desnutrição e surgimento de doenças, principalmente diarréicas e respiratórias 1 . Utilizando apenas a deficiência nutricional, consideraríamos $13,4 \%$ com z escore $<-2$ e $34,7 \%$ com $z$ escore $<-1$ de estatura para idade das crianças em estado de saúde precário; utilizando apenas a deficiência do desenvolvimento físico-motor, 3\%; apenas intercorrências nos últimos trinta dias, $81,7 \%$ (pelo menos uma) e $49 \%$ $(\geq 3)$, valores bastante diferentes entre si. Todavia, a combinação ponderada dessas condições revela 59,4\% das crianças com saúde ruim.

Estudos revelam a importância das condições do ambiente na saúde infantil, apontando que este condiciona a piora, melhora ou manutenção da saúde, excepcionalmente nos dois primeiros anos de vida, momento no qual o ambiente em que se vive age como catalisador do desenvolvimento da criança 1,19 . Neste estudo, observouse que as crianças provenientes de famílias com casa própria têm mais chance de ter saúde ruim 
do que aquelas sem casa própria, condição que aponta a exposição ao ambiente ao qual estão sujeitas. É importante salientar que há certa homogeneidade nas condições de moradia de cada comunidade ribeirinha. Em geral, as casas são muito parecidas, contando com apenas um cômodo, como dormitório.

O significado de ter casa própria em comunidades ribeirinhas representa a manutenção da estória familiar, uma vez que os terrenos são distribuídos na própria família, que já possui a posse deles há muitos anos, e cada novo núcleo familiar constrói a sua casa nesses terrenos. Portanto, casas são alugadas apenas por pessoas de fora da comunidade, que decidiram iniciar nova vida no local. Nesse contexto, a casa própria é mais barata de se morar e representa maior tempo de exposição às adversidades locais, como as precárias condições de moradia, saneamento, assistência à saúde e acesso a alimentos. Ao contrário do que ocorre entre as populações urbanas, nas quais ter casa própria significa melhor condição sócio-econômica, nas comunidades em estudo, a posse da residência representa muito mais a continuidade da exposição à situação ambiental adversa do que proteção. Adicionalmente, a prevalência de saúde ruim aumenta com a idade das crianças, reforçando a hipótese de maior exposição a situações de risco.

Alguns trabalhos avaliaram as condiçõos de moradia, como saneamento básico e características da moradia, e sua influência na saúde da criança. Rissin et al. 20 analisaram as condições da moradia como marcadores de risco nutricional em crianças da área urbana e rural de Pernambuco. Os autores encontraram que, na área rural, o quadro de pobreza é mais generalizado, e as crianças que viviam em domicílios com três ou mais moradores por cômodo e ausência de esgotamento sanitário adequado apresentavam maior risco de deficiência de estatura para idade $(<-2 \mathrm{z}$ escore)

As comunidades ribeirinhas localizadas em áreas rurais estão condicionadas a ambientes de difícil locomoção em virtude das características geográficas, muitas vezes de difícil acesso aos serviços de saúde. A Região Norte, em especial, é caracterizada por extensas áreas de terra e baixa densidade demográfica, dificultando o acompanhamento de saúde adequado e possibilitando maior exposição a doenças infantis. O Fundo das Nações Unidas para a Infância (UNICEF) 2 aponta que a situação da saúde infantil é mais grave em áreas rurais ou em favelas urbanas, onde as mulheres normalmente são analfabetas e enfrentam barreiras sociais que limitam o acesso aos serviços de saúde. Os dados do presente estudo indicam mais de $40 \%$ de mães com baixa escolaridade (0-7 anos de estudo) e mais de $60 \%$ destas não inseridas no mercado de trabalho, o que pode estar associado à menor procura por serviços de saúde e atenção à saúde da criança adequados.

Além das particularidades geográficas, populações ribeirinhas utilizam, comumente, práticas populares em saúde, como chás e benzeduras, alegando que os tratamentos convencionais oferecidos por entidades oficiais de saúde nem sempre correspondem às necessidades da comunidade. Essas práticas inviabilizam a ação e o acompanhamento de equipes de saúde ${ }^{21}$. Em adição, destaca-se que, no cenário amazônico, notam-se maiores concentrações de mães adolescentes, taxas de crescimento populacional em ascendência, baixos índices de urbanização, alto fluxo imigratório e desorganização de serviços de atenção à saúde, com destaque para os serviços destinados à saúde da mulher e da criança 22 .

Fatores sócio-econômicos e demográficos agem diretamente no ambiente, e o local de residência se constitui como fator de risco ou proteção, sendo perceptível a diferença entre área urbana e rural: em meio urbano, as situações de exposição são mais heterogêneas, o contrário do que ocorre no meio rural ${ }^{20}$. As comunidades avaliadas apresentam como característica comum o fato de serem ribeirinhas; portanto, supõe-se que estão expostas aos mesmos fatores de risco ou proteção à saúde das crianças. 


\section{Resumo}

O objetivo foi identificar os determinantes da saúde ruim em populações ribeirinhas menores de dois anos, residentes no Pará, Brasil. Foram avaliadas 202 crianças, considerando-se saúde ruim como variável desfecho, sendo composta pela combinação do estado nutricional, desenvolvimento físico-motor e intercorrências no último mês. Utilizou-se modelo multinível de análise hierárquica, considerando-se como preditoras da saúde ruim variáveis com $p<0,05$ após ajuste. A razão de chance bruta apontou que o estado de saúde ruim é maior para as crianças de famílias que têm casa própria, são de maior idade e têm probabilidade de aleitamento materno exclusivo aos dois, três, quatro e cinco meses. Após ajuste, observa-se que crianças provenientes de famílias com casa própria têm 2,76 vezes mais chance de ter saúde ruim; esta também aumenta com a idade, chegando a ser 5,04 vezes maior entre as crianças de 18 a 23 meses, comparativamente às menores de 7 meses. Ter casa própria e mais idade representam, nessas comunidades, mais tempo de exposição ao risco de saúde ruim.

Saúde da Criança; População Rural; Estado Nutricional; Nível de Saúde

\section{Colaboradores}

S. A. Silva participou do levantamento e análise de dados e da redação do texto. E. C. Moura contribuiu na delimitação da metodologia e revisão do texto.

\section{Agradecimentos}

Agradecemos o financiamento da Secretaria de Ciência Tecnologia e Insumos Estratégicos do Pará (SECTAM/ PA), por meio da Fundação de Amparo à Pesquisa do Estado do Pará (FAPESPA), convênio no. 048/2007.

\section{Referências}

1. Ministério da Saúde. Programas e projetos da saúde da criança: responsabilidades compartilhadas em benefício das crianças brasileiras. Rev Bras Saúde Matern Infant 2002; 2:194-6.

2. Fundo das Nações Unidas para a Infância. Situação mundial da infância 2008: sobrevivência infantil. Brasília: Fundo das Nações Unidas para a Infância; 2007.

3. Onis M, Blössner M. The World Health Organization Global Database on Child Growth and Malnutrition: methodology and applications. Int J Epidemiol 2003; 32:518-26.

4. Departamento de Atenção Básica, Secretaria de Políticas de Saúde, Ministério da Saúde. Saúde da criança: acompanhamento do crescimento e desenvolvimento infantil. Brasília: Ministério da Saúde; 2002.

5. World Health Organization. WHO Child Growth Standards: lenght/height-for-age, weight-for-age, weight-for-lenght, weight-forheight and body mass index-for-age. Methods and development. Geneva: World Health Organization; 2006.
6. Wijnhoven TM, Onis M, Onyango AW, Wang T, Bjoerneboe GE, Bhandari N, et al. Assessment of gross motor development in the WHO Multicentre Growth Reference Study. Food Nutr Bull 2004; 25(1 Suppl):S37-45.

7. Victora CG, Huttly AR, Fuchs SC, Olinto MT. The role of conceptual frameworks in epidemiological analysis: a hierarchical approach. Int J Epidemiol 1997; 26:224-7.

8. Monteiro CA, Benício MHA, Konno SC, Silva ACF, Lima ALL, Conde WL. Causas do declínio da desnutrição infantil no Brasil, 1996-2007. Rev Saúde Pública 2009; 43:35-43.

9. Secretaria de Ciência, Tecnologia e Insumos Estratégicos, Ministério da Saúde. Pesquisa Nacional de Demografia e Saúde da Criança e da Mulher, 2006. Brasília: Ministério da Saúde; 2008.

10. Onis M, Garza C, Victora CG, Onyango AW, Frongillo EA, Martines J. The WHO Multicentre Growth Reference Study: planning, study design, and methodology. Food Nutr Bull 2004; 25(1 Suppl):S15-26. 
11. Engstrom EM, Anjos LA. Déficit estatural nas crianças brasileiras: relação com condições sócioambientais e estado nutricional materno. Cad Saúde Pública 1999; 15:559-67.

12. Instituto Nacional de Alimentação e Nutrição. Pesquisa Nacional sobre Saúde e Nutrição: perfil de crescimento da população brasileira de 0 a 25 anos. Brasília: Instituto Nacional de Alimentação e Nutrição; 1990.

13. Hamill PV, Drizd TA, Johnson CL, Reed RB, Roche AF. NCHS growth curves for children birth-18 years. Vital Health Stat 11 1977; (165):i-iv, 1-74.

14. Telles MS, Macedo CS. Relação entre desenvolvimento motor corporal e aquisição de habilidades orais. Pró-fono 2008; 20:117-22.

15. Prietsch SOM, Fischer GB, Cesar JA, Fabris AR, Mehanna H, Ferreira THP, et al. Doença aguda das vias aéreas inferiores em menores de cinco anos: influência do ambiente doméstico e do tabagismo materno. J Pediatr (Rio J.) 2002; 78:415-22

16. Guarim VLMS. A educação e a sustentabilidade ambiental em comunidades ribeirinhas de Mato Grosso, Brasil. Bol Mus Para Emilio Goeldi, Zool 2005; 1:7-44.
17. Silva GAP, Lira PIC, Lima MC. Fatores de risco para doença diarréica no lactente: um estudo casocontrole. Cad Saúde Pública 2004; 20:589-95.

18. Prietsch SOM, Fischer GB, César JA, Lempek BS, Barbosa Júnior LV, Zogbi L, et al. Doença respiratória em menores de 5 anos no sul do Brasil: influência do ambiente doméstico. Rev Panam Salud Pública 2003; 13:303-10.

19. Willrich A, Azevedo CCF, Fernandes JO. Desenvolvimento motor na infância: influência dos fatores de risco e programas de intervenção. Revista Neurociências 2009; 17:51-6

20. Rissin A, Batista MF, Benício MHDA, Figueiroa JN. Condições de moradia como preditores de riscos nutricionais em crianças de Pernambuco, Brasil. Rev Bras Saúde Matern Infant 2006; 6:59-67.

21. Carreira L, Alvim NAT. O cuidar ribeirinho: as práticas populares de saúde em famílias da ilha Mutum, Estado do Paraná. Acta Sci 2002; 24:791-801.

22. Organização Pan-Americana da Saúde. Epidemiologia das desigualdades em saúde no Brasil: um estudo exploratório. Brasília: Organização PanAmericana da Saúde; 2002.

Recebido em 15/Jun/2009

Versão final reapresentada em 19/Out/2009

Aprovado em 27/Out/2009 